\title{
Instrucción pública y demanda por preceptores normalistas en la Araucanía del siglo XIX ${ }^{1}$
}

\author{
Public instruction and demand for Normal school counselors in the \\ Araucania region during the 19th Century
}
Instrução pública e demanda por preceptores normalistas na Araucanía do século XIX

Omar Turra-Díaz ${ }^{2}$

Universidad del Bío Bío (Chillán-Chile)

Angélica Torres Vásquez ${ }^{3}$

Universidad Católica de Teтuco (Temuco-Chile)

Recepción: 15/12/2016

Evaluación:13/04/2017

Aceptación: 05/05/2017

Artículo de Reflexión

DOI: https://doi.org/10.19053/01227238.6244

\section{RESUMEN}

Este artículo tiene como propósito aportar escuelas, se presentan datos acerca conocimiento acerca del ejercicio docente en la Araucanía, durante la segunda mitad del siglo XIX, en el contexto de una política estatal que buscó expandir la instrucción pública chilena en el territorio Mapuche. En base a información documental, extraída principalmente de los informes de los Visitadores de

de la formación y desempeño de los educadores encargados de impartir las primeras letras en el contexto educativo interétnico.

De acuerdo con la información aportada por las fuentes, la falta de preparación en métodos de enseñanza y la carencia de educadores con estudios formales,

1 El artículo presenta resultados del proyecto interno Grupos de Investigación de la Universidad Católica de Temuco, código DGIPUCT N ${ }^{\circ}$ 2015GE-OT-01.

2 Académico del Departamento de Ciencias de la Educación, Facultad de Educación y Humanidades, Universidad del Bío Bío, Chillán-Chile. Email: oturra@ubiobio.cl

3 Académica de la Facultad de Educación, Universidad Católica de Temuco, Temuco-Chile. Email: atorvas@uct.cl 
constituyó un reclamo persistente de Visitadores y autoridades locales, los que a su vez demandaron la presencia de preceptores normalistas. Desde esta perspectiva el estudio presenta antecedentes que permiten comprender la fundación de Escuelas Normales en el territorio de la Araucanía, desde la última década del siglo XIX.

Palabras clave: Revista Historia de la Educación Latinoamericana, Instrucción Pública, Escuelas de Profesores, Preceptores, Araucanía.

\section{ABSTRACT}

This article aims to provide knowledge about the teaching performance in the Araucanía region, during the second half of the 19th century, in the context of a state policy that sought to expand Chilean public education in Mapuche territory. This research is based on documentary information, extracted mainly from the reports of the visitors to the schools. It is presented here some data about the training and performance of educators responsible for imparting the first letters in the interethnic educational context.

According to the information provided by the sources, the lack of preparation in teaching methods and the lack of educators with formal studies constituted a persistent complaint from Visitors and local authorities, which in turn demanded the presence of Normal school counselors. From this perspective the study expose precedents that allow to understand the foundation of Normal Schools in the territory of the Araucanía, starting over the last decade of century XIX.

Keywords: Journal History of Latin American Education, Public Instruction, Schools of Teachers, counselors, Araucanía.

\section{RESUMO}

Este artigo tem como propósito produzir conhecimentos acerca do exercício docente na Araucanía, durante a segunda metade do século XIX, no contexto de uma política estatal que buscou expandir a instrução pública chilena no território Mapuche. Com base em informação documental, extraída principalmente dos informes dos Visitadores de escolas, são apresentados dados acerca da formação e desempenho dos educadores encarregados de lecionar as primeiras letras no contexto educacional interétnico. De acordo com a informação fornecida pelas fontes, a falta de preparação em métodos de ensino e a carência de educadores com estudos formais constituíram uma reinvindicação persistente de Visitadores e autoridades locais, os que a sua vez demandaram a presença de preceptores normalistas. A partir desta perspectiva, o estudo apresenta antecedentes que permitem compreender a fundação de Escolas Normais no território da Araucanía, desde a última década do século XIX.

Palavras-chave: Revista História da Educação Latino-americana, Instrução Pública, Escolas de Professores, Preceptores, Araucanía. 


\section{INTRODUCCIÓN}

La formación del profesorado chileno en una institución especializada se puede representar como un fenómeno educativo de larga tradición en el contexto latinoamericano. Su origen se remonta a la década del 40' del siglo XIX, cuando un conjunto de condiciones culturales y políticas permitieron la fundación de la primera institución formadora de docentes en el país. Con la creación de la Escuela Normal de Preceptores en 1842 y de Preceptoras en 1854, ambos establecimientos localizados en Santiago, la capital de la república, se inauguró un modelo de formación de educadores y educadoras de instrucción primaria de rigurosa preparación e influencia cultural en el país ${ }^{4}$.

Mientras se formaban los preceptores normalistas, el oficio docente era desempeñado por personas que por diversas motivaciones se dedicaban a la enseñanza, pero que carecían de una formación especializada en sus métodos. En su mayoría, eran miembros de la comunidad que habían cursado estudios elementales y al dedicarse a la enseñanza en escuelas primarias aseguraban un sueldo estatal, que si bien era precario, se recibía con regularidad.

En la época, esto ocurría en todo el país, aunque en algunos territorios adquiría dimensiones específicas según la relación política y cultural establecida con el estado chileno. Un caso particular en este sentido fue la Araucanía ${ }^{5}$, el territorio habitado por el pueblo originario Mapuche, que se enfrentó primero a las huestes coloniales españolas y luego con las fuerzas militares chilenas. ${ }^{6}$ Durante la segunda mitad del siglo XIX, tierras y fronteras indígenas fueron paulatinamente estrechándose hasta quedar definitivamente ocupadas por el estado nacional a fines del período. ${ }^{7}$ El avance sobre el territorio Mapuche estuvo marcado por un profundo proceso de transformación sociocultural generado tanto por las dinámicas propias de las relaciones sociales interétnicas resultantes del contacto entre los habitantes originarios y la población inmigrante, como por los

4 Iván Núñez, “Las Escuelas Normales: Una historia de fortalezas y debilidades 1842-1973”, Docencia, no. 40 (2010): 34.

5 Los conquistadores españoles denominaron araucanos al pueblo mapuche y, por consecuencia, Araucanía al territorio que habitaban. Se trata de un territorio que se extendía desde el rio Bío Bío por el norte hasta el seno del Reloncaví por el sur, en el centro-sur del Chile actual.

6 El territorio Mapuche del período histórico, aquel que comienza con la llegada de los españoles, comprendía un amplio espacio geográfico que se localiza en la parte sur del actual territorio chileno, entre el río Bío Bío y el archipiélago de Chiloé. Al respecto véase José Manuel Zavala, Los Mapuches del siglo XVIII. Dinámica interétnica y estrategias de Resistencia (Santiago: Editorial Universidad Bolivariana, 2008).

7 El proceso de avance y ocupación del territorio Mapuche por el estado chileno puede verse, entre otros, en los siguientes estudios: Arturo Leiva, El primer avance a la Araucanía: Angol 1862 (Temuco: Universidad de la Frontera, 1984); Jorge Pinto, De la inclusión a la exclusión. La formación del estado, la nación y el pueblo Mapuche (Santiago: Colección IDEA/USACH, 2000); José Bengoa, Historia del pueblo Mapuche (siglo XIX y XX) (Santiago: Ediciones LOM, 2000); Leonardo León, Araucanía: la violencia mestiza y el mito de la Pacificación, 1880-1900 (Santiago: Editorial ARCIS, 2005). 
dispositivos y mecanismos estatales que aportaron a la configuración de una nueva trama sociocultural en el territorio, entre ellos, la creación de centros urbanos, vías de transporte y de comunicación, y el establecimiento de una red de escuelas públicas que tenía como propósito alfabetizar a la población Mapuche y no Mapuche del territorio ocupado.

En el extenso territorio Mapuche, las relaciones coloniales configuraron dos modelos de educación con fines específicos y diferenciados, los que se desarrollaron de manera paralela en un contexto de relaciones interétnicas. Para el caso de la sociedad Mapuche, el programa formativo organiza el conocimiento, los saberes educativos a transmitir, de acuerdo a las edades y el género y las formas de enseñar y aprender en la comunidad, es decir, orienta el proceso de formación de persona que la sociedad Mapuche construyó para formar a sus nuevas generaciones y garantizar su proyección como sociedad y como pueblo. ${ }^{8}$ Evidentemente es un programa educativo sin escuela -en el entendido que esta institución es de origen occidentalpero que da cuenta del horizonte conceptual y principios educativos que organizaban el proceso de enseñanza y aprendizaje en la sociedad Mapuche.

Por su parte, el asentamiento español en los márgenes de la Araucanía conllevó el inicio del modelo educacional occidental, basado en la enseñanza de la lectura y escritura castellana, entre las comunidades Mapuche. Este modelo educacional asociado a la estrategia de evangelización que estaba a la base de la conquista española en América, fue desarrollado por sacerdotes pertenecientes a congregaciones religiosas que llegaron con los primeros conquistadores ${ }^{9}$. El programa formativo buscaba la asimilación y aculturación de los jóvenes mapuche a la cultura hispano-occidental, por lo que su enseñanza se concretaba con la iniciación de los educandos indígenas en la doctrina católica y en la castellanización de su lenguaje.

El Estado chileno replicó el modelo de las escuelas misionales en la Araucanía, luego del proceso independentista, siendo la evangelización y castellanización la base del programa formativo pero ahora con el propósito de integrar al pueblo Mapuche a la civilización y a la chilenidad. No obstante, la convivencia interétnica y proyección de los programas formativos experimentó un drástico revés histórico, hacia fines de la centuria decimonónica, cuando el Estado chileno emprende la ocupación material de la Araucanía.

8 Son los propios cronistas españoles del siglo XVII y XVIII quienes testimoniaron la existencia de un conjunto de saberes, conocimientos y destrezas como constituyentes de un itinerario formativo de los infantes y jóvenes mapuches, así como unas formas de enseñar y aprender que se diferenciaban según el género y la edad. Al respecto véase Desiderio Catriquir y Teresa Durán, "Inagekey mapunche rakiduam. Algunos principios educativos mapunche" (Temuco: UCT-Documento de Trabajo, 1993).

9 Jorge Pinto, Misioneros en la Araucanía, 1600-1900: un capítulo de historia fronteriza en Chile (Bogotá: CELAM, 1990). 
Desde la mirada intracultural Mapuche ${ }^{10}$, se ha planteado que con la ocupación militar de la Araucanía y el avance reduccional, se paralelizaron dos modelos formativos de educadores que expresaban lógicas de comprensión del mundo diferenciadas y desde las cuales, ambas sociedades, promovieron la distribución de su conocimiento y acervo cultural; el Mapuche tradicional representado en los kimeltuchefe ${ }^{11}$, y el chileno-occidental representado en los preceptores. Se trata de dos agentes formadores que respondían a racionalidades culturales y orientaciones educativas distintas, pero que en sus ámbitos de actuación fueron altamente valorados por su rol de alfabetizadores socioculturales y por el dominio de unos métodos de enseñanza que eran el resultado de una rigurosa formación.

Por su parte, la historia de la educación en Chile ha planteado que la instrucción pública que promovió el Estado decimonónico en la Araucanía tuvo como objetivo fundamental actuar como mecanismo de cohesión y control social para la conformación de una nueva sociedad ${ }^{12}$, para cuyos fines la red de escuelas establecidas en el territorio se configuró como un verdadero dispositivo simbólico orientado a 'civilizar' y chilenizar a la sociedad y cultura Mapuche. ${ }^{13}$

Respecto de la formación del profesorado normalista en Chile, la investigación educativa existente no es especialmente abundante y tampoco ha abordado este tipo de formación docente como un objeto de estudio sistemático y abarcativo en su desarrollo histórico. Sin embargo, los escasos estudios existentes permiten dimensionar la importancia educativa y social del normalismo en el país. Así, la investigación de Muñoz y Ebensperguer ${ }^{14}$ aporta información detallada acerca de la distribución de las Normales en el territorio nacional, de su organización administrativa y de los planes formativos, en las primeras décadas del siglo XX. Peña, ${ }^{15}$ a través del estudio de la Escuela Normal de Preceptoras de Santiago, configura una visión del normalismo decimonónico desde la perspectiva femenina. Por su parte, el

10 Dejamos constancia que desde hace un buen tiempo (1993), en escritos académicos producidos en perspectiva intracultural, se utiliza el concepto Mapunche como forma de rescatar la autodenominación ancestral del pueblo que hoy se conoce como Mapuche, nominación otorgada por la sociedad hispano-criolla y luego oficializada por el Estado Chileno. Al respecto véase Desiderio Catriquir y Teresa Durán, documento de trabajo arriba citado, y Desiderio Catriquir y Gabriel Llanquinao, "Educación intercultural en contexto interétnico: formación y desempeño docente, Anthropos, no. 207 (2005), 167-184.

11 Denominación que se otorga a los educadores encargados de transmitir el mapuche kimün (conocimiento) en la sociedad mapuche.

12 Véase Sol Serrano, "De escuelas indígenas sin pueblos a pueblos sin escuelas indígenas: La educación en la Araucanía en el siglo XIX”, Historia, no. 29, (1995-1996), 423-474; Andrés Donoso, Educación y nación al sur de la frontera. Organizaciones mapuche en el umbral de nuestra contemporaneidad, 1880-1930 (Santiago: Ediciones Pehuén, 2007).

13 Omar Turra Díaz, "De la ocupación a la aculturación: escuela chilena y pueblo Mapuche en los siglos XIX y XX”, Revista de Historia 18, no. 1 (2008), 29-42.

14 Gertrudis Muñoz de Ebensperguer, "El desarrollo de las Escuelas Normales en Chile", Anales de la Universidad de Chile, no. 45-46 (1942), 152-186.

15 Macarena Peña Tondreau, "Hijas Amadas de la Patria. Historia de la Escuela Normal de Preceptoras de Santiago, 1854-1883" (tesis Licenciatura en Historia, Pontificia Universidad Católica de Chile, 2000). 
estudio de Nuñez ${ }^{16}$ presenta a las Escuelas Normales desde una mirada de síntesis, mostrando su devenir histórico a la base de sus fortalezas y debilidades; otra investigación de Núñez ${ }^{17}$, provee información sobre sus actores -alumnos, directores y profesores-y reflexiona sobre sus aportes y problemáticas en la historia larga de estas instituciones, hasta su supresión en el año 1973.

Pero es el estudio de Serrano, Ponce de León y Rengifo ${ }^{18}$ el que aborda al preceptorado como actor social en el siglo XIX, relevando su heterogeneidad en cuanto a formación y la precariedad económica desde la que desempeñaban su labor educativa.

No obstante, los estudios indicados tienen su foco de interés en las instituciones formadoras y la situación del preceptorado, que se desempeñaba en el centro administrativo del país y en las regiones aledañas. Por lo mismo, poco y nada se sabe acerca del ejercicio docente en el singular contexto educativo, social y cultural que representaba la Araucanía en el proceso de formación de estado. ¿Quiénes ejercían la función docente? ¿Cómo se valoraba/cuestionaba su desempeño educativo en las aulas?, son preguntas que pretendemos abordar en este artículo. Para ello utilizamos una metodología de carácter histórico, en base a dos fases principales: la heurística, por la cual se seleccionaron y organizaron las fuentes documentales de época. Al respecto, los documentos principales que aportan información al estudio son los Informes emitidos por los Visitadores de escuelas al Ministerio ${ }^{19}$, y otros Informes generados por autoridades locales preocupados por la instrucción pública; la hermenéutica, entendida como construcción de sentido en base a procesos interpretativos realizados desde los propósitos del estudio y con soporte en las fuentes.

El artículo expone como uno de sus principales resultados, la demanda persistente por la presencia de preceptores con formación institucionalizada, es decir, preceptores normalistas, por lo que puede representarse como un estudio de los antecedentes que permiten comprender la fundación de Escuelas Normales en el territorio de la Araucanía, desde la última década del siglo XIX.

\footnotetext{
16 Iván Núñez Prieto, “Las Escuelas Normales: una historia de fortalezas y debilidades 1842-1973”, Docencia, no. 10 (2010), 32-39.

17 Iván Núñez Prieto, "Escuelas Normales. Una historia larga y sorprendente. Chile (1842-1973)", Revista Pensamiento Educativo, no. 46-47 (2010), 133-150.

18 Sol Serrano, Macarena Ponce de León y Francisca Rengifo, Historia de la Educación en Chile (1810 - 2010), tomo II (Santiago: Taurus, 2012), 253- 289.

19 La organización e impulso de la instrucción pública en el país dependía del Ministerio de Justicia, Culto e Instrucción Pública.
} 


\section{La preocupación por la instrucción primaria popular}

La necesidad de conformar un sistema educativo bajo control estatal es una preocupación planteada desde los inicios de la República, pero es recién en la década de 1840 que se concretó su organización. Este sistema educacional surge segmentado en dos ámbitos o subsistemas de acuerdo a los requerimientos de la estructura social binaria de la época, la mayoría popular y la élite aristocrática. Ignacio Domeyko, interesado en la promoción educativa en el país, da cuenta de esta situación hacia 1842 cuando plantea que:

"habiendo en Chile como en todas las naciones del mundo dos clases...que son: $1^{\circ}$ La clase pobre que vive del trabajo mecánico de sus manos y a la cual ni el tiempo, ni el retiro en que vive, ni sus hábitos, costumbres y ocupaciones permiten tomar parte efectiva en los negocios públicos aunque la Constitución les diese facultades para esto. $2^{\circ}$ La clase que desde la infancia se destina para formar el Cuerpo Gubernativo de la República. La instrucción pública debe también dividirse en dos ramos que son: Instrucción primaria para la primera; Instrucción superior para la segunda clase"20.

Uno de estos subsistemas, es el conformado por la educación secundaria y universitaria, teniendo por características el ser altamente selectivo, crecer de "arriba hacia abajo" y contar con el apoyo mayoritario de intelectuales y políticos de la época. ${ }^{21}$ El otro, refiere al subsistema primario dirigido a los sectores populares, cuya base de apoyo se encuentra en una minoría liberal que comprende la educación popular como un motor de progreso social y económico. ${ }^{22}$

Sin embargo, el desarrollo de la instrucción primaria popular no ocurre por oposición a las élites gobernantes sino que, como plantea el historiador Gabriel Salazar, fue funcional al proyecto político y económico de la clase mercantil dirigente. ${ }^{23}$ En sus palabras, la responsabilidad estatal por la educación primaria emergió

"impulsado por la necesidad de activar el disciplinamiento moral de 'los que vivían del trabajo material de sus manos', a objeto de integrarlos funcionalmente al orden mercantil nacional, pero sin alterar su condición de clase ni su (eventual) rol económico" 24 .

20 Citado por Arturo Mancilla. "Antecedentes para una historia de la educación primaria en Chile. Siglos XIX y comienzos del XX". (Tesis para optar al grado de Magíster en Historia. Universidad de Chile, 2005), 32.

21 Cristian Cox y Jacqueline Gysling, La formación del profesorado en Chile, 1842-1987 (Santiago: CIDE, 1990), 35.

22 A mediados del siglo XIX, ya se encuentra organizado por estamentos sociales la función educativa de las escuelas de primeras letras. A la escuela fiscal o municipal, escuela pública, asisten los hijos e hijas de labradores, artesanos o peones, mientras que los hijos e hijas de los sectores acomodados desarrollaban sus estudios en establecimientos particulares o en la preparatoria de los institutos.

23 Gabriel Salazar, “Los dilemas históricos de la auto-educación popular en Chile. ¿Integración o autonomía relativa?", Proposiciones, No, 15 (1988): 84-129.

24 Ibídem, 91 
La conformación del sistema de educación primaria estatal/popular fue resultado de una labor complementaria entre intelectuales que demandaron esta educación y gobernantes que prestaron interés a estas ideas para implementarlas como política pública. Los llamados educacionistas del siglo XIX, en particular Domingo Faustino Sarmiento y los hermanos Gregorio y Miguel Luis Amunátegui, proporcionaron los fundamentos que relevaron la importancia de la instrucción primaria para la modernización de la sociedad los que, al poco tiempo, se tradujeron en la creación de instituciones educativas y en cuerpos legales que vinieron a sentar las bases de este tipo de educación, como es la Ley de Instrucción Primaria de 1860.

Sarmiento expuso sus ideas en el escrito titulado 'De la Educación Popular' (1849), en cuyas páginas constataba el atraso científico y tecnológico heredado de la colonización española y afirmaba que la grandeza de una nación dependía de la capacidad industrial, intelectual y moral de sus habitantes, siendo responsabilidad de la instrucción primaria pública desarrollar esas capacidades, pues

la concurrencia de los niños a la escuela, trae el efecto moralizador de absorver una parte de tiempo, que sin ella sería disipado en la ociosidad, i en abandono; habituar el espíritu a la idea de un deber regular, continuo, le da lo que es hábitos de regularidad en sus operaciones...echa sin sentirlo los primeros rudimentos de moralidad $i$ de sociabilidad tan necesarios, para prepararlos a las obligaciones i deberes de la vida de adultos ${ }^{25}$.

Para los Amunátegui, quienes expresaron sus ideas en la obra 'De la instrucción primaria en Chile; lo que es, lo que debería ser' (1856), el fomento de la educación del pueblo reportaba una serie de beneficios a la República, entre otros: actuar como mecanismo de moralización y de formación de virtudes en los individuos,

la asistencia sola a la escuela donde se enseñan la lectura i escritura, i la disciplina que se observa en ella, propenden activamente a la educación del corazón de los alumnos. Los niños, jeneralmente hablando, contraen en la escuela hábitos de orden, de sumisión, de trabajo continuado e incesante, que más tarde no pueden olvidar. En el taller, o en cualquiera otra parte, desplegarán las mismas virtudes que en la escuela. El alumno acostumbrado a llenar sus deberes con exactitud...será con toda probabilidad un individuo honrado, que no faltará nunca a su palabra, que ejecutará sus obras con método... ${ }^{26}$;

Impulsaba el desarrollo económico con la preparación de trabajadores inteligentes para la industria y la agricultura,

25 Domingo Faustino Sarmiento, De la Educación Popular (Santiago: Imprenta de Julio Belín y Compañía, 1849), 37-38.

26 Miguel Luis Amunátegui y Gregorio Víctor Amunátegui, De la Instrucción Primaria en Chile: lo que es, lo que debería ser (Santiago: Imprenta del Ferrocarril, 1856), 20. 
La industria no florece sino en los países civilizados... Un pueblo estúpido i grosero puede producir cargadores, esto es, bestias humanas capaces de trasportar los fardos más pesados, o peones...pero no esos obreros intelijentes a quienes la instrucción primaria comunica la fuerza a la par que la destreza...La fundación de escuelas es la manera más eficaz de que surjan las fábricas y los talleres"... "Si Chile quiere el progreso de su agricultura $i$ de su industria...es preciso que difunda la instrucción primaria por todos los ángulos de su territorio ${ }^{27}$;

también beneficia la participación y estabilidad política con su aporte a la conformación de ciudadanos letrados, pues

la instrucción pública es un elemento de orden, una garantía de estabilidad, una prenda segura de acierto. Los gobiernos están tan penetrados de esta verdad, que en casi todas las constituciones han impuesto la educación pública como una obligación del Estado, la cualidad de saber leer $i$ escribir como uno de los requisitos esenciales para ser ciudadano ${ }^{28}$.

La preocupación por la educación primaria se relacionaba con los imperativos de civilización y moralización del pueblo, componentes de relevancia en los procesos de modernización que impulsaba la élite gobernante. Para ello, el aumento de los niveles de instrucción elemental era considerado como una condición de la modernización y un requerimiento para el ingreso a los mercados internacionales. ${ }^{29}$

Por otra parte, la expansión del sistema educacional primario como parte del proyecto liberal republicano buscaba la formación de una comunidad política cada vez más extensa, compuesta por ciudadanos comprometidos e integrados social y culturalmente a la República. Es decir, el desarrollo de la instrucción primaria vendría a aportar a la conformación de un sujeto productivo a la vez que un sujeto político.

Estas razones que fundamentaban la necesidad de expandir la instrucción primaria en el país, sin duda estaban presentes en el ideario de los educacionistas cuando miraban hacia el sur, hacia la Araucanía, aunque para este territorio todas estas aspiraciones se complejizaban producto del "estado de atraso" del componente humano que lo habitaba: el pueblo Mapuche. Condición que Vicente Fidel López, en el primer Manual de Historia de Chile publicado en 1846, se preocupó de precisar identificando en Chile la presencia de

dos razas distintas; la primera es aqella de qe nosotros formamos parte $i$ qe llamamos Chilenos, en la acepción estricta de la palabra; la otra es la de los indios, qe aunque son chilenos también, porque han nacido en el territorio de Chile i porque

27 Ibídem, 40, 50.

28 Ibídem, 64.

29 María Loreto Egaña, La educación primaria popular en el siglo XIX en Chile: Una práctica de política estatal (Santiago: LOM Ediciones, 2000), 13. 
poseen una gran parte de él, no son miembros de nuestra sociedad...nosotros ablamos español y vivimos civilizadamente... No siendo los indios ombres de nuestra raza ni de nuestras costumbres, debemos ver que su historia no es la nuestra: además, siendo bárbaros, debemos creer qe no tienen historia ${ }^{30}$.

Como en los tiempos coloniales, intelectuales y gobernantes vieron en la educación un medio eficaz para transformar al mapuche en un sujeto civilizado y con ello avanzar en la ocupación de su territorio. ${ }^{31}$ No obstante, un intelectual como Ignacio Domeyko, quien visitó el territorio Mapuche para luego publicar la obra La Araucanía y sus habitantes (1846), se oponía con firmeza a cualquier estrategia de ocupación militar depositando su confianza en la labor educativa que desarrollarían los misioneros. ${ }^{32}$ En efecto, en un primer momento, la función educativa se confirió a los misioneros religiosos que llegaron al territorio, los franciscanos en 1837 y capuchinos en 1849, quienes vinieron a proyectar en la república la escuela misional colonial. Se partía del supuesto que la labor educativa de la escuela misional traería como "consecuencia inevitable la insensible y pacífica destrucción de la barbarie" como planteaba en 1849 el Ministro de Justicia, Culto e Instrucción Pública. ${ }^{33}$

Sin embargo, a poco andar la escuela misional es puesta en entredicho, pues para las autoridades la formación en rudimentos de la fe cristiana parecía insuficiente y debía complementarse con enseñanzas de escritura y lectura en español, además de nociones de aritmética. En todo caso su presencia en el territorio se consideraba de utilidad, pues además de aportar a la educación, contribuía a evitar levantamientos y a aglutinar población alrededor de las misiones, considerándoseles verdaderos centinelas de la civilización entre los indígenas.

La escuela pública en la Araucanía comenzó a extenderse luego de 1850, primero en territorios cristianizados, más tarde con el avance de la línea de la frontera y colonización chilena en el territorio. Estas escuelas se establecieron en los pueblos luego de la petición de los vecinos, colonos chilenos e inmigrantes, y la posterior decisión administrativa que aprobaba su financiamiento, por lo que la escuela pública en el territorio mapuche era hija de los pueblos y desde ahí su pretensión era "difundir las luces entre los indígenas vecinos de esos pueblos" ${ }^{34}$ Luego de la ocupación del territorio Mapuche por el Estado chileno, la escuela y la instrucción pública pasaron

30 Vicente Fidel López, Manual de la Historia de Chile. Libro adoptado por la Universidad para la enseñanza en las Escuelas de la República (Santiago: Imprenta del Progreso, 1846), 25-26.

31 En base a este ideario se configuró la llamada educación asimilacionista como un primer modelo de entendimiento y gestión social de la diversidad cultural indígena. Desde sus preceptos la realidad multicultural queda subsumida en una voluntad integradora promovida por la cultura europeo-occidental, que se autoimpone como superior, a través de una praxis educativa impregnada de etnocentrismo.

32 Ignacio Domeyko, Araucanía y sus habitantes: recuerdos de un viaje hecho en las provincias meridionales de Chile en los meses de enero $y$ febrero de 1845 (Santiago: Imprenta Chilena, 1846).

33 Citado por Serrano, op. cit., 432.

34 Este es uno de los planteamientos centrales de la historiadora Sol Serrano en el estudio que venimos citando, 454. 
a tener un rol relevante en el propósito de integrar al pueblo indígena a la sociedad chilena por lo que se intensificó la creación de escuelas en pueblos y villas que se habían ido fundando con el avance militar en la Araucanía. En el ánimo de civilizar al indígena y cambiar sus costumbres, quedaba de plano descartado una educación específica dirigida a la población Mapuche en sus nuevos espacios de reducción. Por tanto, el acceso e influencia de la doctrina cristiana y primeras letras castellanas quedó reducida a un grupo minoritario de educandos mapuches que asistían a la escuela chilena en los pueblos.

Como el programa formativo de la escuela chilena no se correspondía con los fines y principios educativos de la sociedad Mapuche su presencia en el territorio indígena resultaba controversial y, por cierto, resistida por parte de sectores y comunidades defensoras de los patrones culturales ancestrales. La enseñanza escolar se asociaba a la imposición de normas y hábitos foráneos, además de un espacio de negación del mapunzugun, la lengua originaria Mapuche. Desde esta comprensión, la escuela era vista como un espacio de perdición del ser Mapuche y por lo tanto de necesaria impugnación y repudio. ${ }^{35}$

\section{El ejercicio del preceptorado en la Araucanía}

A mediados del siglo XIX, las autoridades gubernamentales en el propósito de organizar y homogeneizar la enseñanza primaria popular, tomaron la decisión de nombrar personas competentes para que visitaran las escuelas de la república e informaran detalladamente acerca de aspectos administrativos y pedagógicos. Surgió entonces la figura de los Visitadores, empleados fiscales dependientes del Ministerio de Instrucción, a quienes se atribuyó la función específica de operativizar la política educativa estatal en el contexto real de la escuela primaria. ${ }^{36} \mathrm{En}$ su labor se entrecruzaban acciones de control administrativo, inspeccionando la aplicación de los reglamentos y disposiciones para la práctica educativa emanada desde el Ministerio, y de modelaje pedagógico, que se traducía en una práctica docente directa en las escuelas visitadas, como manera de inducir y uniformar metodologías de enseñanza en los educadores primarios. ${ }^{37} \mathrm{Y}$ aquello podían realizarlo desde su experiencia formativa, pues los Visitadores habían cursado estudios

35 Pablo Marimán, “Demanda por educación en el movimiento mapuche en Chile 1910-1995”, en Pueblos indígenas: educación y desarrollo, eds. Álvaro Bello, Pablo Marimán y Angélica Wilson (Temuco: Instituto de Estudios Indígenas, 1997),130-200.

36 De acuerdo con el Informe elaborado por el Ministro de Justicia, Culto e Instrucción Pública, en julio de 1853, "para uniformar la enseñanza bajo los mejores métodos e introducir en las escuelas arreglos convenientes $i$ un nuevo espíritu que las saque del abatimiento en que están muchas de ellas" tres visitadores recorrían por aquel entonces diversos puntos de la república, implementando mejoras en los establecimientos primarios y levantando una completa relación estadística que el Ministro adjuntaba en su informe. En: El Monitor de las Escuelas Primarias, Archivo Histórico Nacional, Tomo 2, No 1, 15 de agosto de 1853, 19.

37 Egaña, op cit. 
y egresado, algunos con altos reconocimientos, de la Escuela Normal de Preceptores de Santiago.

Si bien, en un primer momento, era reducido el número de estos funcionarios su importancia educativa llevó a que el Estado progresivamente aumentara su dotación. ${ }^{38}$ Sus visitas y los correspondientes informes que emitían, dejaban en evidencia las deplorables condiciones en que se desenvolvía la educación primaria decimonónica, en dimensiones como infraestructura, mobiliario, recursos didácticos, asistencia escolar y metodologías de enseñanza, entre otras. También, exponían la situación de los funcionarios que actuaban como preceptores, quienes en su gran mayoría no habían sido formados en una Escuela Normal, sino que eran simplemente estudiantes aventajados que se habían legitimado en sus comunidades para desempeñar la función docente.

Durante la segunda parte del siglo XIX los preceptores, en la república, constituían un grupo de educadores cuya característica era su heterogeneidad. ${ }^{39}$ Entre ellos, era posible distinguir a "los normalistas", educadores y educadoras que habían realizado sus estudios en una Escuela Normal, "los propietarios", que si bien no habían asistido a una Normal rindieron un examen de acuerdo a las exigencias de la misma; y "los interinos" que realizaban docencia sin haber rendido el examen que los habilitara como propietarios. El aumento de las escuelas primarias y el reducido contingente de preceptores normalistas que egresaban a la época, generó un protagonismo educativo creciente de los preceptores interinos, por sobre los propietarios y los normalistas. Así, del 20\% que representaban en 1880, pasaron a un $68 \%$ en $1897 . .^{40}$

Para el caso de la Araucanía, su especificidad histórica permitía el protagonismo pedagógico, además, de otro educador; el sacerdote de la escuela misional. De origen europeo, estos educadores desarrollaban una enseñanza, fundamentalmente, de la doctrina cristiana. Los visitadores fueron críticos de la enseñanza de estas escuelas, pues los sacerdotes/preceptores eran de edad avanzada y desconocían los nuevos métodos pedagógicos.

De acuerdo con la información aportada por los Visitadores, el ejercicio docente en la Araucanía era desarrollado mayoritariamente por un preceptorado interino, que carecía de idoneidad y entusiasmo para la labor formativa. Según Informe del Visitador de escuelas de la Provincia de Arauco, para el año 1855

Puede decirse de un modo general que la instrucción primaria solo principia a nacer en el Departamento de que me ocupo. La carencia de preceptores idóneos y

38 El número de estos funcionarios "en terreno" siempre fue escaso en consideración a las trece provincias del país a la época, lo que explica su paulatino crecimiento: de tres en 1853 su número se duplicó al año siguiente y para 1855 ya eran 8 los visitadores. Posteriormente, la Ley de Instrucción Primaria de 1860, consideró la asignación de un visitador por cada provincia del territorio. Véase Emilio Ramirez, "Niños populares y escuela popular: un estudio sobre las dificultades del proceso de escolarización en Chile (1840-1860)". Polis. Revista Latinoamericana 13, no. 37 (2014): 167-189.

39 Al respecto véase Sol Serrano, Macarena Ponce de León y Francisca Rengifo, Historia de la Educación en Chile (1810 - 2010), tomo II (Santiago: Taurus, 2012).

40 Ibídem, 253. 
entusiastas... las faltas de locales i útiles para la escuela, han sido a mi ver los motivos que las han tenido en un estado de abyección ${ }^{41}$.

En un Informe posterior se precisa mejor el predominio de los interinos respecto de los normalistas y su efecto sobre la enseñanza,

Atendiendo a la preparación normal solo hai en la provincia 34 maestros que han sido graduados en la Escuela Normal i que, por lo tanto, gozan de una buena preparación profesional. El resto, es decir, 113 empleados son interinos... los profesores más idóneos son contados en esas rejiones... apenas llegan al $24 \%$ del personal, número más que insuficiente para poder realizar la reforma que debe imprimirse en nuestro sistema de enseñanza ${ }^{42}$.

Crecientemente, el predominio de un preceptorado carente de formación regular será objeto de cuestionamiento por los informes que se emitían a las autoridades educativas del nivel central. Uno de los primeros que se pronunció sobre el particular fue José Bernardo Suárez, un respetado preceptor, quien luego de recorrer en el año 1854 escuelas de Valdivia y la misión de Trumag, en el Departamento de La Unión, en su informe emitido a la autoridad educativa, reivindicaba la presencia de una escuela con preceptores medianamente preparados,

...mas no hablo de escuelas como las que nominalmente existen en las misiones; hablo de establecimientos dirigidos por preceptores medianamente idóneos, en que se eduque al niño indígena y se le enseñe la doctrina cristiana, la lectura, la escritura y las cuentas". 43

Al decir de Suárez, Valdivia era la provincia del país más atrasada en instrucción, pues estimaba la existencia en su territorio de 4.000 niños indígenas en edad de recibir instrucción primaria que no sabían de escuela.

En el mismo sentido, puede leerse el informe del visitador de la provincia de Concepción, frontera norte de la Araucanía, el que comunicaba en su Memoria anual que

la gran cantidad de empleados interinos, no idóneos para desempeñar el cargo de preceptorado $i$ por su falta de preparación i conocimiento del nuevo sistema de enseñanza, no ha permitido al infraescrito obtener los frutos que esperaba, sino en parte... ${ }^{44}$.

41 Informe del Visitador de Los Ángeles, Manuel Valenzuela, visitando escuelas de la Provincia de Arauco, específicamente del Departamento de Laja. El Monitor de las Escuelas Primarias, Biblioteca Nacional de Chile, Tomo III, No 9, 15 de junio de 1855, 270.

42 Informe sobre las escuelas públicas al sur de Concepción, presentado al Sr. Inspector General de Instrucción Primaria por el Visitador Extraordinario don Ruperto Oroz, 18 de agosto de 1897. Archivo Nacional de Chile. Ministerio de Educación, vol.: 1191, Instrucción Primaria, Notas de Talca a Magallanes, 1897, 239.

43 El Monitor de las Escuelas Primarias. Biblioteca Nacional de Chile, Tomo II. N 11, 15 junio 1854, 290.

44 Memoria anual del visitador de escuelas de Concepción correspondiente al año 1899, Concepción, enero de 1900. El Educador 
La preparación en métodos de enseñanza y, particularmente, el nuevo sistema hacía referencia a la incorporación de la pedagogía alemana en la formación normalista ${ }^{45}$, de fines del siglo XIX, y es algo que resienten los Visitadores pues los educadores interinos estaban lejos de conocer y menos de introducir en sus escuelas este sistema de enseñanza. La mayor parte de los preceptores no normalistas no comprendían toda la importancia de un buen sistema de enseñanza, ni poseían los menores conocimientos en pedagogía.

En este contexto la presencia de preceptores competentes o idóneos, representaba una necesidad para avanzar en la expansión de la instrucción pública en el territorio y, para algunos, el mecanismo simbólico pertinente para introducir a los indígenas en el camino de la civilización y la moralidad.

\section{La demanda por preceptores normalistas}

La formación de preceptores en Escuelas Normales tuvo como objetivo fundamental el aportar al desarrollo de idoneidad y moralidad de quienes desempeñarían la función docente en las escuelas de instrucción primaria del país. La idoneidad se vinculaba al dominio de métodos de enseñanza y en la apropiación de los conocimientos elementales de las materias a enseñar, es decir, refiere a una formación en conocimientos fundamentales de pedagogía. La moralización, por su parte, se identificaba con la formación en buenas costumbres y un comportamiento de acuerdo a hábitos civilizados. Los civilizadores de la masa popular, tenían que ser sujetos civilizados ${ }^{46}$.

El problema al respecto, era que las Escuelas Normales creadas en el siglo XIX formaban cohortes de preceptores que no alcanzaban a cubrir las necesidades del sistema de instrucción primaria. Por un lado, la tasa de egresados era baja y por otro el número de escuelas públicas fue paulatinamente creciendo lo que generaba una desproporción entre los requerimientos del sistema escolar y la disponibilidad de preceptores normalistas. La necesidad de contar con educadores que se hicieran cargo de las escuelas fundadas, concedía protagonismo a preceptores improvisados o interinos.

Como se ha indicado más arriba, en la Araucanía la labor educativa era desempeñada en su mayoría por preceptores interinos, cuya idoneidad en el trabajo pedagógico resultaba una fuente de cuestionamiento por parte de los Visitadores, los que también identificaban esta falta de idoneidad con el estado de atraso y el mal funcionamiento de la instrucción pública

Penquista. Biblioteca Nacional de Chile, Año 1, $\mathrm{N}^{\circ}$ 3, 4 y 5, 1 de abril de 1900.

45 En 1885 llegan preceptoras y preceptores alemanes a introducir el nuevo sistema de enseñanza en las Escuelas Normales, el que se denominaba "método intuitivo" y consistía en reemplazar al aprendizaje memorístico por un aprendizaje razonado y psicológicamente conducido, a través de diversas etapas. Buscaba también colocar a los aprendices frente a realidades concretas.

46 Núñez, op cit., 35. 
en el territorio. Es por ello que la demanda por preceptores normalistas en los diversos informes adquiría cada vez más notoriedad y persistencia.

Las fuentes nos permiten plantear que además del problema de la idoneidad docente, también se cuestionaba ciertos comportamientos personales reñidos con la labor formadora, es decir con problemas asociados a la moralidad docente. Un caso que ilustra esta situación es el comunicado por el Gobernador de Angol al Ministro de Instrucción Pública, en carta fechada el año 1886, en que expone la petición hecha a él por el comandante de la plaza de Temuco respecto del cuestionable desempeño del preceptor de la escuela de niños de aquella localidad.

Verá usted -comunicaba el Gobernador-que el preceptor que la rejenta, don Silvestre Díaz, ha estado mui lejos de satisfacer medianamente sus deberes pues el lamentable estado de atraso en que se hallan sus discípulos es debido a su poca constracción por ser demasiado aficionado a la vevida (escrita con $v$ en el original) del licor, vicio que tiende a desmoralizar al que está encargado de dirijir; por esta consideración me permito indicar a Ud que se hace urjente su reemplazo ${ }^{47}$.

En la parte final de su exposición el Gobernador Gorostiaga planteaba al Ministro la conveniencia de separar al preceptor de su puesto y nombrar "cuanto antes un normalista que le reemplace".

Otra situación, que se puede asociar a descuido o incumplimiento administrativo, es el denunciado por el visitador de Arauco en su Informe dirigido al Inspector General de Instrucción Pública, el año 1898, en el que exponía el mal proceder del preceptor de la escuela de hombres № 1 de Cañete, don Obligado Vera, por no presentar a sus estudiantes a examen ante la comisión nombrada para el efecto y comunicada con anterioridad. Indicaba el visitador que no había causa alguna que justifique semejante irregularidad, menos aún que el preceptor mencionara como razón que era el día viernes después de mediodía, y en esa hora las escuelas urbanas no funcionaban.

Junto a la denuncia de esta irregularidad, se consignaba un atraso escolar evidente en los estudiantes, por lo que el visitador recomendaba que

sería un beneficio para la instrucción en el pueblo de Cañete si se pidiera separación o traslado a otra parte don Obligado Vera i que la escuela que actualmente rejenta fuera encomendada a un normalista moderno que pudiera elevarla al nivel de las más adelantadas de la provincia ${ }^{48}$.

47 Archivo Nacional de Chile. Ministerio de Educación, v. 624, Instrucción Pública Gobernación de Angol, 1886 a 12 de Abril de 1887.

48 Archivo Nacional de Chile. Ministerio de Educación, vol. 1121, Instrucción Pública. Decretos Na 330 a 400, 1898, 100-101. 
Desde los requerimientos de Visitadores y autoridades, la presencia de preceptores normalistas en las escuelas del territorio garantizaba idoneidad y moralidad para el desempeño docente, por lo que no se reservaban juicios para recomendar y solicitar que estos docentes se hicieran cargo de las escuelas, que en un número creciente, se fundaban en la Araucanía luego de su ocupación territorial. De seguro, estas mismas peticiones recibidas en el Ministerio, influyeron para que este organismo impulsara la creación de la primera Escuela Normal en la Araucanía, la que fue establecida en Valdivia el año 1896. A esta inicial fundación, se agregaron otras tres escuelas normales estatales, a inicios del siglo XX, localizadas en distintos centros urbanos del antiguo territorio mapuche (Puerto Montt, Angol y Victoria).

Es evidente que desde los testimonios de Visitadores y autoridades la especificidad del contexto Mapuche, su diversidad cultural y lingüística, no aparece como un requerimiento de idoneidad para el desempeño docente, por lo que resulta de interés indagar en las actas de fundación de estas Escuelas Normales, en sus fines y propósitos, y en el programa formativo que estaba a su base, de modo de reconocer su aporte a la conformación de una educación monocultural/intercultural en el contexto interétnico. Aquellos son desafíos investigativos que se abordarán en el futuro inmediato.

\section{CONCLUSIÓN}

La ocupación territorial de la Araucanía por parte del estado chileno, significó para la sociedad Mapuche la pérdida de su autonomía política-cultural y, con ello, el comienzo de un proceso de forzada aculturación. Las nuevas condiciones sociopolíticas, pérdida de soberanía y agrupamiento en reducciones, generaron un repliegue del programa formativo Mapuche a una esfera privada, al espacio familiar inmediato, configurándose como una instancia de oposición y/o resistencia al modelo educativo oficial representado por la escuela chilena.

Como la escuela, en el contexto interétnico, se justificaba en base a propósitos civilizadores y asimiladores a la chilenidad, quedaba descartado cualquier atisbo de una educación dirigida a la población Mapuche en su especificidad cultural y lingüística.

De esta manera, en el contexto de una política estatal que buscaba expandir la instrucción pública en el territorio Mapuche, el oficio docente en la Araucanía fue desempeñado, mayoritariamente, por personas que carecían de una formación pedagógica institucionalizada. Se trataba de preceptores interinos o improvisados que, por pertenecer a las comunidades en que se establecían las escuelas y tener cursado estudios elementales, asumían la función docente.

Desde los informes realizados por los Visitadores se cuestionaba, con habitualidad, la labor educativa de estos preceptores por su falta de idoneidad para el trabajo pedagógico, también por su 'moralidad', situaciones que se 
asociaban con el estado de atraso y mal funcionamiento de la instrucción pública en escuelas de la Araucanía.

En este contexto, la presencia de preceptores competentes o idóneos formados en escuelas normales se configuró como una demanda educativa de creciente explicitación en los documentos que se emitían al Ministerio de Instrucción Pública. Sin embargo, una formación en los saberes y formas culturales Mapuche no aparece como un requerimiento de idoneidad para el desempeño docente.

De esta forma, la conjunción de factores como la expansión de la red de escuelas públicas, la demanda por preceptores con formación idónea y el consenso por promover la educación en el territorio Mapuche ocupado/colonizado, convirtieron en necesidad el disponer de centros de formación de preceptores en el espacio local, por lo que las autoridades gubernamentales dieron carta de aprobación a la creación de Escuelas Normales, en diversas localidades de la Araucanía, desde fines del período en estudio.

\section{FUENTES}

Archivo Nacional de Chile (AN), Santiago - Chile. Ministerio de Educación (MEDU), Fondo Instrucción Primaria.

Biblioteca Nacional de Chile (BN), Santiago - Chile. Sección Hemeroteca, Fondo Bibliográfico sala Medina. El Monitor de las Escuelas Primarias.

Biblioteca Nacional de Chile (AN), Santiago - Chile. Colección Digital. El Educador Penquista.

\section{REFERENCIAS}

Amunátegui, Miguel Luis y Amunátegui, Gregorio Víctor. De la Instrucción Primaria en Chile: lo que es, lo que debería ser. Santiago: Imprenta del Ferrocarril, 1856.

Bengoa, José. Historia del pueblo mapuche (siglo XIX y XX). Santiago: Ediciones LOM, 2000.

Catriquir Colipán, Desiderio y Durán, Teresa. Inagekey mapunche rakiduam. Algunos principios educativos Mapunche. Documento de Trabajo, Universidad Católica Temuco, 1993.

Catriquir Colipán, Desiderio y Llanquinao, Gabriel. "Educación intercultural en contexto interétnico: formación y desempeño docente". Revista Anthropos. No. 207 (2005): 167-184.

Cox, Cristian y Gysling, Jacqueline. La formación del profesorado en Chile, 1842-1987. Santiago: CIDE, 1990. 
Domeyko, Ignacio. Araucanía y sus habitantes: recuerdos de un viaje hecho en las provincias meridionales de Chile en los meses de enero y febrero de 1845. Santiago: Imprenta Chilena, 1846.

Donoso, Andrés. Educación y nación al sur de la frontera. Organizaciones mapuche en el umbral de nuestra contemporaneidad, 1880-1930. Santiago: Pehuén, 2007.

Egaña, María Loreto. La educación primaria popular en el siglo XIX en Chile: Una práctica de política estatal. Santiago: LOM Ediciones, 2000.

Leiva, Arturo. El primer avance a la Araucanía: Angol 1862. Temuco: Universidad de la Frontera, 1984.

León, Leonardo. Araucanía: la violencia mestiza y el mito de la Pacificación, 1880-1900. Santiago: Editorial ARCIS, 2005.

López, Vicente Fidel. Manual de la Historia de Chile. Libro adoptado por la Universidad para la enseñanza en las Escuelas de la República. Santiago: Imprenta del Progreso, 1846.

Mancilla, Arturo. "Antecedentes para una historia de la educación primaria en Chile. Siglos XIX y comienzos del XX". Tesis de Magíster en Historia, Universidad de Chile, 2005.

Marimán, Pablo. “Demanda por educación en el movimiento mapuche en Chile 19101995". En Pueblos indígenas: educación y desarrollo, editado por Álvaro Bello, Pablo Marimán y Angélica Wilson. Temuco: Instituto de Estudios Indígenas, 1997, 130200.

Muñoz de Ebensperguer, Gertrudis. "El desarrollo de las Escuelas Normales en Chile". Anales de la Universidad de Chile, No. 45-46 (1942): 152-186.

Núñez Prieto, Iván. “Las Escuelas Normales: Una historia de fortalezas y debilidades 1842-1973". Revista Docencia. No. 4 (2010): 32-39.

Núñez Prieto, Iván. “Escuelas Normales. Una historia larga y sorprendente. Chile (18421973)". Revista Pensamiento Educativo, No. 46-47 (2010): 133-150.

Peña Tondreau, Macarena "Hijas Amadas de la Patria. Historia de la Escuela Normal de Preceptoras de Santiago, 1854-1883". Tesis Licenciatura en Historia, Pontificia Universidad Católica de Chile, 2000.

Pinto, Jorge. Misioneros en la Araucanía, 1600-1900: Un capítulo de historia fronteriza en Chile. Bogotá: CELAM, 1990.

Pinto, Jorge. De la inclusión a la exclusión. La formación del estado, la nación y el pueblo mapuche. Santiago: Colección IDEA/USACH, 2000.

Ramírez, Emilio. “Niños populares y escuela popular: un estudio sobre las dificultades del proceso de escolarización en Chile (1840-1860)". Polis. Revista Latinoamericana, 13. No. 37 (2014): 167-189.

Salazar, Gabriel. "Los dilemas históricos de la auto-educación popular en Chile. ¿Integración o autonomía relativa?". Revista Proposiciones. No. 15 (1988): 84-129.

Sarmiento, Domingo Faustino. De la Educación Popular. Santiago: Imprenta de Julio Belín y Compañía, 1849. 
Serrano, Sol. "De escuelas indígenas sin pueblos a pueblos sin escuelas indígenas: La educación en la Araucanía en el siglo XIX". Revista Historia. No. 29 (1995-1996): 423-474.

Serrano, Sol, Ponce de León, Macarena y Rengifo, Francisca. Historia de la Educación en Chile (1810 - 2010), tomo II. Santiago: Taurus, 2012.

Turra Díaz, Omar. “De la ocupación a la aculturación: escuela chilena y pueblo mapuche en los siglos XIX y XX". Revista de Historia, 18. No. 1 (2008): 29-42.

Zavala, José Manuel. Los mapuches del siglo XVIII. Dinámica interétnica y estrategias de Resistencia. Santiago: Editorial Universidad Bolivariana, 2008.

\begin{tabular}{l|l|} 
& \\
\hline & Turra-Díaz, Omar; Torres Vásquez, Angélica. “Instrucción pública \\
y demanda por preceptores normalistas en la Araucanía del siglo & \\
XIX”. Revista Historia de la Educación Latinoamericana. Vol. 19 No. \\
28 (2017): 129-148
\end{tabular}




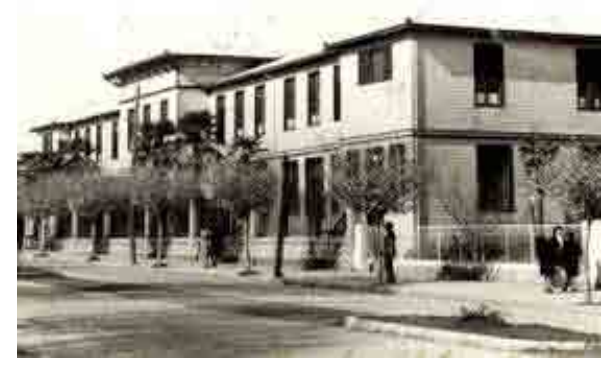

Fuente: Escuela Normal de Angol, región de la Araucanía, fundada en junio de 1904. 